Int. J. Odontostomat.,

4(2):105-110, 2010.

\title{
Dientes Natales, Revisión Bibliográfica y Caso Clínico
}

\author{
Natal Teeth, Case Report and Review
}

\author{
Claudia Fierro Monti"; Lorena Bravo Rivera ${ }^{\star *}$; Francisca Torres Chianale ${ }^{\star *}$; Camila Álvarez Helle ${ }^{\star \star \star}$ \& Ma \\ Antonieta Pérez Flores ${ }^{* * * *}$
}

FIERRO, M. C.; BRAVO, R. L.; TORRES C. F.; ÁLVAREZ, H. C. \& PÉREZ, F. M. A. Dientes natales, revisión bibliográfica y caso clínico. Int. J. Odontostomat., 4(2):105-110, 2010.

RESUMEN: Los dientes natales y neonatales deben valorarse con mucho cuidado; estimando su movilidad, integridad y la presencia de una úlcera (Riga Fede) en la superficie ventral de la lengua causada por su roce con el diente. Los dientes natales podrían parecerse a la dentición temporal normal en tamaño y forma, sin embargo, también pueden presentarse más pequeños, cónicos, con esmalte y dentina hipoplásicos, con poca formación o ausencia de sus raíces. La mayoría de estos dientes no son supernumerarios y no se requieren extracciones en todos los casos. Es de fundamental importancia el seguimiento periódico de un odontopediatra. En este artículo se presenta un caso clínico y se revisa la epidemiología, etiología, presentación clínica, diagnóstico diferencial, así como, el manejo de los dientes natales.

PALABRAS CLAVE: dientes natales, dientes neonatales.

\section{INTRODUCCIÓN}

La odontogénesis es un proceso complejo en el que están implicados el ectodermo, el mesodermo y células procedentes de la cresta neural. Dentro de este proceso, la Erupción Dentaria se define como el movimiento de un diente de su sitio de desarrollo, dentro del proceso alveolar hasta su posición funcional en la cavidad bucal (Schour \& Massler, 1941).

Tradicionalmente, se denomina erupción dental al momento eruptivo en que el diente rompe la mucosa bucal y hace su aparición en la boca del niño. Según Barbería (2001) este concepto es erróneo, ya que en el sentido más estricto, la erupción dental, dura toda la vida del diente, comprende diversas fases e implica el desarrollo embriológico de los dientes, los movimientos de desplazamiento y el acomodo en las arcadas. La aparición del diente en la boca recibe el nombre de emergencia dentaria y, aunque es llamativo para el niño, sólo constituye uno de los parámetros para la evaluación de la normalidad o no del proceso.
El proceso de erupción cumple con las siguientes observaciones:

1. Los dientes se mueven en el espacio tridimensionalmente, no sólo a lo largo de su eje.

2. Los dientes erupcionan con características diferentes y velocidades, específicas en cada etapa.

3. Los dientes llegan a una posición funcional que es hereditaria (Marks \& Schroeder, 1996).

Los dientes temporales comienzan su emergencia entre los 6 y 9 meses de vida. Los primeros suelen ser los incisivos inferiores y los últimos los segundos molares temporales. Se acepta que en la dentición temporal la variabilidad en la cronología y la secuencia es muy amplia, hasta el punto de tomarse como normal si al cumplir los 3 años de vida todos los dientes han hecho emergencia, independientemente del momento y el orden (Barbería).

Prof Asociado, Depto. Pediatría Bucal Facultad de Odontología, Universidad de Concepción. Especialista en Odontopediatría, UDEC, Chile.

** Instructor, Depto. Pediatría Bucal Facultad de Odontología, Universidad de Concepción. Especialista en Odontopediatría, UDEC

*** Alumna Pregrado, Facultad de Odontología, Universidad de Concepción, Chile.

${ }^{* * * *}$ Prof. Asistente, Magister Odontopediatría, Universidad Complutense, Madrid. Depto. Pediatría Bucal, Facultad de Odontología Universidad de Concepción, Chile. 
Aunque la erupción de los incisivos deciduos inferiores, al nacer, es normal en muchos mamíferos, los dientes natales son raros en humanos (Leung, 1986b).

La clasificación, de Massler \& Savara (1950) para los dientes que erupcionan prematuramente es una de las más utilizadas donde los dientes presentes al nacimiento son llamados dientes natales, mientras que aquellos que erupcionan treinta días después del nacimiento son llamados dientes neonatales. Esta terminología únicamente diferencia el periodo de aparición de los dientes en la cavidad oral. Mientras otro autores como Spouge \& Feasby (1966) sugirieron que estos dientes podrían clasificarse de mejor forma según su grado de madurez, siendo un diente natal o neonatal, maduro aquel que tiene un desarrollo normal y que tiene relativamente buen pronóstico; el término inmaduro de un diente natal o neonatal implica un desarrollo defectuoso con un mal pronóstico en cuanto a la retención en la cavidad oral.

Los dientes natales son generalmente una problema benigno. Sin embargo, pueden interferir con la lactancia materna y, si están sueltos o móviles pueden ser tragados o aspirados durante la lactancia" (Cunha et al., 2001).

\section{Prevalencia}

La incidencia de los dientes natales oscila entre 1:2.000 a 1:3.500 nacidos vivos. Kates et al. (1984) observaron 18.155 recién nacidos con una prevalencia de 1:716. Leung (1986a) observó 50.892 partos (durante 17 años) y encontró que la incidencia de los dientes natales era 1:3.392. Con respecto a la distribución por género algunos investigadores como Kates et al. observaron una mayor prevalencia de dientes natales en las mujeres con un $66 \%$. Los dientes natales son raros en los infantes extremadamente prematuros (Sureshkumar \& McAulay, 2002).

En el caso de la alta prevalencia en niños con fisura labio platina publicada por De Almeida \& Gomide (1996), los autores lo atribuyen a la alteración dental del alvéolo en la fisura y a la posición superficial del diente en esta región. Hay una variación racial en la incidencia, ya que el problema es más común entre los niños de algunas tribus Indio Americanas (Leung, 2006a). Al parecer la variación en la prevalencia depende de la población estudiada y a los métodos empleados en cada estudio.

\section{Etiología}

La presencia de dientes natales y neonatales definitivamente es una alteración cronológica e histológica cuya etiología todavía es desconocida. Y han sido involucrados como factores causantes la posición superficial del germen dentario, las infecciones, los estados febriles, los traumatismos, la desnutrición, la estimulación hormonal y la exposición materna a toxinas ambientales (Gladen et al., 1990; Alaluusua et al., 2002). La condición puede ocurrir como un rasgo familiar ya que una historia familiar positiva ha sido obtenida entre el $8-62 \%$ de los casos (Zhu \& King, 1995). Se ha sugerido también la transmisión hereditaria de un gen autosómico dominante (Cunha et al.). Los dientes natales se presentan en el $2 \%$ de los niños con fisura labial y palatina unilateral y el $10 \%$ de los niños con fisuras bilaterales (De Almeida \& Gomide). Los dientes natales han sido notificados en asociación con síndromes como el Ellis-van Creveld (displasia condroectodérmica), JadassohnLewandowsky (Paquioniquia congénita), El síndrome de Hallerman-Streiff (oculomandibulofacial con hipotricosis), Disostosis craneofacial, steacystoma multiplex, Sotos, Wiedemann-Rautenstrauch, MeckelGruber y Pierre Robin (Marakoglu et al., 2004).

\section{Características clínicas}

Los dientes más comúnmente afectados son los incisivos centrales inferiores (85\%), seguido de los incisivos superiores $(11 \%)$, los caninos y molares inferiores $(3 \%)$, y los caninos y molares maxilares, $(1 \%)$ (Zhu \& King). La fuerte predilección por los incisivos centrales inferiores es coherente con el orden normal de erupción de los dientes temporales. Los dientes natales usualmente se observan en pares (Cunha et al.). La erupción de más de dos dientes natales es poco frecuente. Masatomi et al. (1991) informaron de un niño con 14 dientes natales. La mayoría de los dientes natales representan la erupción temprana de la dentición primaria normal. Menos del $10 \%$ es supernumerario (Adekoya-Sofowora, 2008). Los dientes natales podrían parecerse en tamaño y forma a los dientes primarios, sin embargo, a menudo son más pequeños, cónicos, de color amarillento, con esmalte y dentina hipoplásicos, presentando el desarrollo de la raíz pobre o ausente (Galassi et al., 2004). La displasia del esmalte está relacionada con la duración de la cubierta gingival. La mayoría de los dientes natales son móviles (Kates, 1984).

La apariencia de cada diente natal puede ser 
clasificada en una de las siguientes cuatro categorías, las cuales están basadas en las observaciones de autores previos (To, 1991; Hebling et al., 1997).

1. Estructura de la corona débilmente unida al alvéolo por un anillo de mucosa oral; sin raíz.

2. Corona sólida débilmente unida al alvéolo por la mucosa oral; pequeña o ninguna raíz.

3. El borde incisal de la corona erupcionó atravesando la mucosa oral.

4. Mucosa oral inflamada con el diente sin erupcionar pero palpable.

Un examen radiográfico es indicado por la literatura para diferenciar la erupción prematura de un diente de la fórmula temporal de uno supernumerario. Idealmente, este estudio debería realizarse en el período de recién nacido. La radiografía también proporciona información acerca del desarrollo de la raíz del diente, la relación entre el diente natal y los dientes adyacentes, y el estado de estructuras del esmalte, y la dentina radicular (Cunha et al.).

La alteración de tejidos blandos más frecuente durante la erupción de los dientes primarios se conoce como hematoma de la erupción. Clínicamente, se presenta como una elevación de la mucosa de coloración azul sobre el alvéolo de un diente próximo a erupcionar. Esta lesión se forma por el acúmulo de sangre alrededor del folículo dental previo a la erupción (Barbería).

\section{Características Histológicas}

A pesar de la estructura básica normal de lo dientes natales, su erupción temprana se asocia con alteraciones la mineralización del esmalte. Histológicamente, la mayoría de los dientes natales han presentado displasias o hipomineralización del esmalte, así como dentina irregular y osteodentina en los cuellos, y dentina interglobular en las regiones de la corona. El borde incisal puede carecer de esmalte. Tanto la vaina Hertwig y el cemento pueden estar ausente. A menudo hay un aumento en el número de vasos sanguíneos dilatados en el tejido pulpar, la formación de la raíz es a menudo incompleta (Seminario \& Ivancakovà, 1992).

\section{Diagnóstico Diferencial}

El diagnóstico diferencial más importante se obtiene mediante una radiografía para diferenciar un diente de la serie normal de un supernumerario, ade- más, se debe diferenciar de lesiones frecuentes en el recién nacido como: Nódulos de Bohn, Perlas de Epstein y Quistes de la lámina dental.

- Perlas de Epstein: Se localizan en la línea media en el paladar. Son quistes de inclusión epitelial.

- Nódulos de Bohn: Localizados en las superficies bucal y lingual del alvéolo. Son restos de muco-tejido de las glándulas mucosas ectópicas.

- Quistes de la lámina dental: Se localizan en la cresta del alvéolo. Son remanentes de la lámina dentaria y están formados por una cavidad quística llena de queratina (Revuelta, 2009).

\section{Manejo Clínico}

Las complicaciones que se pueden derivar de la presencia de los dientes natales son la aspiración o deglución de los dientes, la laceración de los senos de la madre, y la ulceración sublingual (Riga-Fede) con la consecuente evitación de la alimentación (AdekoyaSofowora).

Si el diente no interfiere con la lactancia materna y en general es asintomático, no es necesario intervenir (Seminario \& Ivancakovà). La extracción del diente se indica si el diente es supernumerario o si el diente no está bien implantado y excesivamente móvil, lo que se asocia con un riesgo de aspiración. Es muy recomendable la consulta con un especialista, fundamentalmente si la extracción de los dientes está en consideración. La extracción del diente debe ir seguida de un curetaje de la cavidad para evitar que células poco desarrolladas de la papila dental y aquellas de la vaina radicular de Hertwig que fácilmente se desprenden de la parte calcificada del diente al permanecer en el alvéolo puedan continuar desarrollándose en una estructura semejante a un diente (Zhu \& King). La falla del curetaje podría resultar en la erupción de los restos odontogénicos que requieran futuro tratamiento. (Ziai et al., 2005)

Si los dientes deben extraerse, es aconsejable esperar a que el lactante tenga por lo menos 10 a 14 días de nacido, esto permitirá que la flora intestinal del lactante produzca vitamina $\mathrm{K}$ la cual es esencial para los niveles adecuados de protrombina que interviene en los procesos de coagulación (Thomas \& Larry, 1985).

Retardar los procedimientos quirúrgicos en los recién nacidos hasta después de los 10 días post-parto no se considera rutinariamente necesario cuando la 
administración profiláctica de vitamina $\mathrm{K}$ es el procedimiento estándar de los hospitales (Holder \& Ashcraft, 1980). Si es necesario, la hemostasis puede lograrse utilizando agentes hemostáticos tópicos en combinación con presión directa (Zhu \& King).

La lesión de Riga-Fede no es una indicación de extracción. El tratamiento de elección de esta úlcera incluye suavizar bordes incisales ásperos o la colocación de resina compuesta sobre los bordes incisales. La extracción temprana de un diente natal primario podría ocasionar apiñamiento de los sucesores permanentes, ya que el espacio originalmente ocupado por los dientes extraídos puede cerrarse y ser ocupado por los dientes vecinos (Goho, 1996).

Procedimiento que puede ser evitable. Se informó sobre 72 dientes natales de los cuales 56 dientes fueron extraídos, y no mostraron pérdida de espacio a causa de la exodoncias. Sin embargo los dientes que no fueron extraídos mostraron desarrollo de la raíz (To).

En este artículo se presenta un caso clínico y se revisa la epidemiología, etiología, presentación clínica, complicaciones así como, el manejo de los dientes natales.

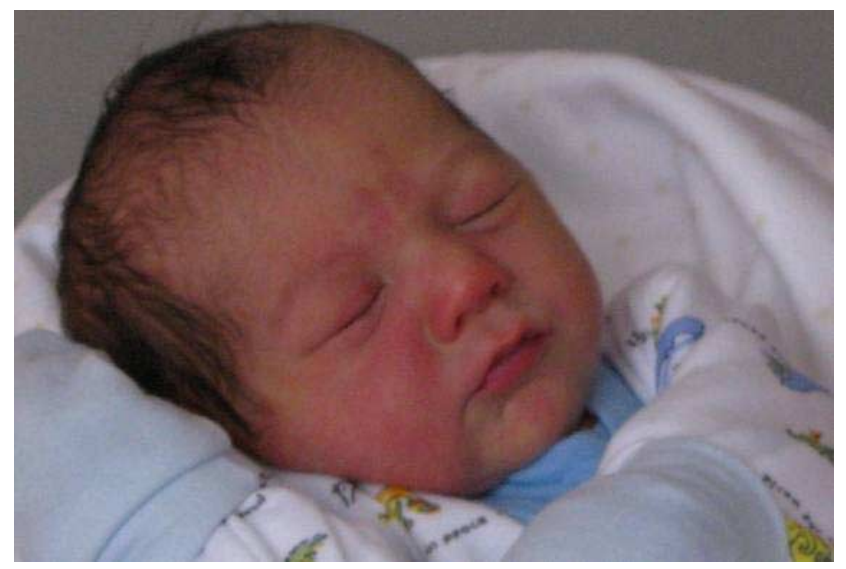

Fig. A Neonato.

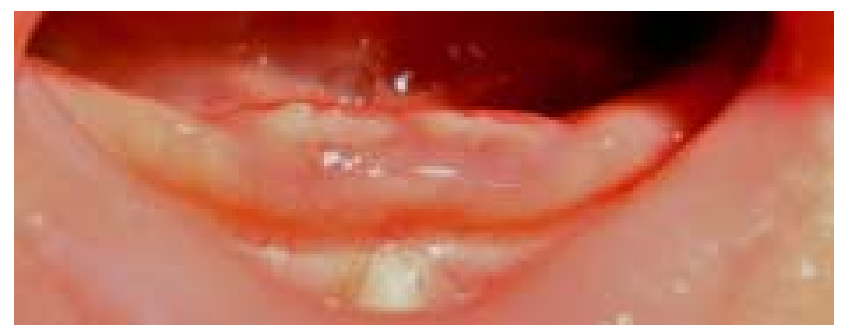

Fig C. Dientes natales.

\section{CASO CLÍNICO}

Se presenta el caso de un recién nacido con dientes natales: Neonato sano, bajo lactancia materna, nacido por parto distósico, sin antecedentes familiares de dientes natales o neonatales, de género masculino.

Examen extraoral: Proporciones faciales, musculatura Facial, y simetría facial, normales (Fig. 1A).

Examen intraoral: Mucosa bucal: normal, 2 dientes natales: incisivos centrales inferiores, emergiendo el borde incisivo, sin movilidad (Fig. 1B,C,D).

\section{CONCLUSIÓN}

Los dientes natales son generalmente una problema benigno. Sin embargo, pueden interferir con la lactancia materna. La extracción del diente se indica si es supernumerario, no está bien implantado o es excesivamente móvil, lo que se asocia con un riesgo de aspiración. Es muy recomendable la consulta con un especialista, fundamentalmente si la extracción de los dientes está en consideración.

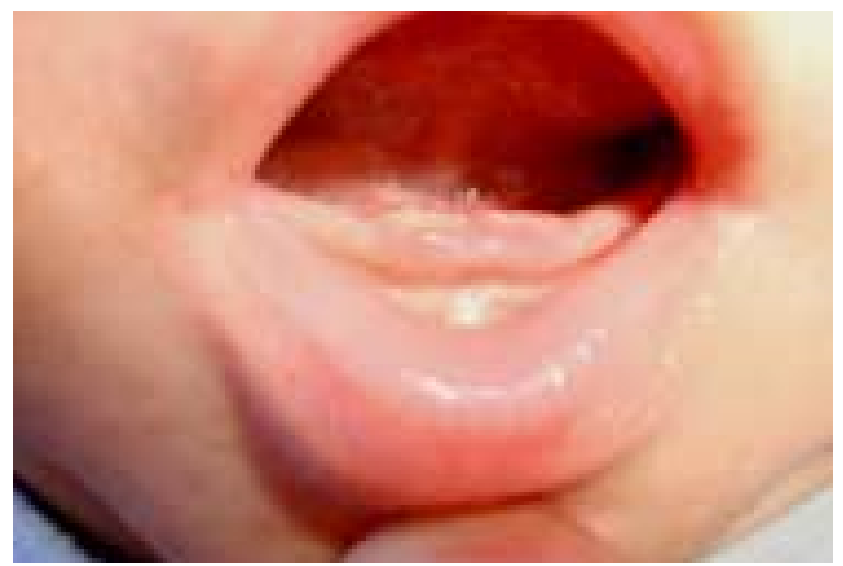

Fig.B Cavidad oral.

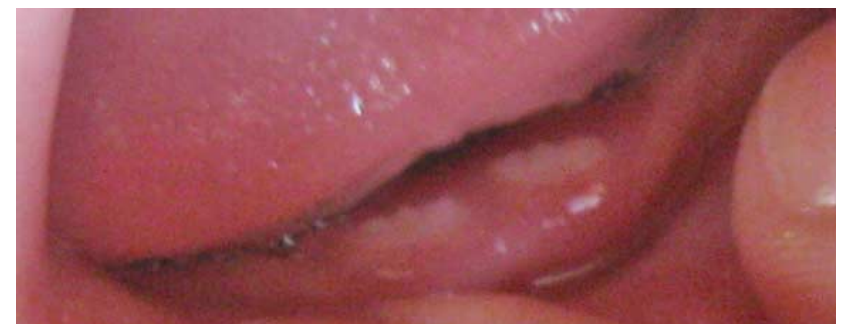

Fig. D. Control 1 mes. 
FIERRO, M. C.; BRAVO, R. L.; TORRES C. F.; ÁLVAREZ, H. C. \& PÉREZ, F. M. A. Natal teeth, case report and review. Int. J. Odontostomat., 4(2):105-110, 2010.

ABSTRACT: Natal and neonatal teeth should be evaluated carefully, considering their mobility, integrity and the presence of an ulcer (Riga Fede) on the ventral surface of the tongue caused by his brush with the tooth. Natal teeth may resemble normal primary dentition in size and shape, however, may also smaller, conical, with hypoplasic enamel and dentin, with little development or absence from their roots. Most of these teeth are not temporary and does not require removal in all cases. It is essential periodic monitoring of a pediatric dentist. This article presents a case report and review the epidemiology, etiology, clinical presentation, differential diagnosis and the management of natal teeth.

KEY WORDS: natal teeth, neonatal teeth.

\section{REFERENCIAS BIBLIOGRÁFICAS}

Adekoya-Sofowora, C. A. Natal and neonatal teeth: a review. Niger Postgrad. Med. J., 15(1):38-41, 2008.

Alaluusua, S.; Kiviranta, H.; Leppäniemi, A.; Hölttä, P.; Lukinmaa, P. L.; Lope, L.; Järvenpää, A. L.; Renlund, M.; Toppari, J.; Virtanen, H.; Kaleva, M. \& Vartiainen, T. Natal and neonatal teeth in relation to environmental toxicants. Pediatr. Res., 52(5):6525, 2002.

Barbería, L. E. Erupción dentaria. Prevención y tratamiento de sus alteraciones. Pediatr. Integral, 6(3):229-40, 2001.

Cunha, R. F.; Boer, F. A. C.; Torriani, D. D. \& Frossard, W. T. Natal and neonatal teeth: review of the literature. Pediatr. Dent., 23:158-62, 2001.

De Almeida, C. M. \& Gomide, M. R. Prevalence of natal/neonatal teeth in cleft lip and palate infants. Cleft Palate Craniofac. J., 33:297-9, 1996.

Galassi, M. S.; Santos-Pinto, L. \& Ramalho L. T. Natal maxillary primary molars:case report. J. Clin. Pediatr. Dent., 29:41-4, 2004.

Gladen , B. C.; Taylor, J. S.; Wu, Y. C.; Ragan, N. B.; Rogan, W. J. \& Hsu, C. C. Dermatological findings in children exposed transplacentally to heatdegradated polychlorinated biphenyls in Taiwan. $\mathrm{Br}$. J. Dermatol., 122:799-808, 1990.

Goho, C. Neonatal sublingual traumatic ulceration (Riga-Fede disease): reports of cases. J. Dent. Child., 63:362-4, 1996.

Hebling, J.; Zuanon, A. C. C. \& Vianna, D. R. Dente Natala case of natal teeth. Odontol Clin., 7:37-40, 1997.
Holder, T. M. \& Ashcraft, K. W. Textbook of Pediatric Surgery. Philadelphia, W. B. Saunders, 1980.

Kates, G. A.; Needleman, H. L. \& Holmes, L. B. Natal and neonatal teeth: a clinical study. J. Am. Dent. Assoc., 109:441-3, 1984.

Leung, A. K. Natal teeth in American Indians. Am. J. Dis. Child., 140:1214, $1986 a$.

Leung, A. K. Natal teeth. AMA Am. J. Dis. Child., 140:249-51, $1986 b$.

Marakoglu, K.; Percin, E. F.; Marakoglu, L.; Gursoy, U. K. \& Goze, F. Anencephalic infant with cleft palate and natal teeth: a case report. Cleft Palate Craniofoc. J., 41:456-8, 2004.

Marks, S. C. Jr, \& Schroeder, H. E. Tooth eruption: theories and facts. Anat. Rec., 245(2):374-93, 1996.

Masatomi, Y.; Abe, K. \& Ooshima, T. Unusual multiple natal teeth: casereport. Pediat. Dent., 13:170-2, 1991.

Massler, M. \& Savara, B. S. Natal and neonatal teeth. A review of 24 cases reported in the literature. $J$. Pediatr., 36:349-59, 1950.

Revuelta, R. La cavidad bucal del nacimiento a la infancia: Desarrollo, patologías y cuidados. Perinatol. Reprod. Hum., 23(2):82-9, 2009.

Schour , I. \& Massler, M. Development of the human dentition. J. Am. Dent. Assoc., 28:1153-60, 1941.

Seminario, A. L. \& Ivancakovà, R. Natal and neonatal teeth. Acta Medica, 47:229-33, 2004. 
FIERRO, M. C.; BRAVO, R. L.; TORRES C. F.; ÁlVAREZ, H. C. \& PÉREZ, F. M. A. Dientes natales, revisión bibliográfica y caso clínico. Int. J. Odontostomat., 4(2):105-110, 2010

Spouge, J. D. \& Feasby, W. H. Erupted teeth in the newborn. Oral Surg. Oral Med. Oral Pathol., 22:198208, 1966.

Sureshkumar, R. \& McAulay, A. H. Natal and neonatal teeth. Arch. Dis. Child. Neonatal Ed., 87:F227, 2002.

To, E. W. A study of natal teeth in Hong Kong Chinese. Int. J. Poediatr. Dent., 1:73-6, 1991.

Thomas, K. B. \& Larry, S. L. Odontología Pediátrica. México, D.F., Manual Moderno, 1985.

Zhu, J. \& King, D. Natal and neonatal teeth. ASDC J. Dent. Child., 62:123-8, 1995.

Ziai, M. N.; Bock, D. J.; Do Silveira, A. \& Daw, J. L. Natal teeth: a potential impediment to nasoalveolar molding in infants with cleft lip and palate. J. Craneofac. Surg., 16:262-6, 2005.
Dirección para correspondencia:

Prof. Dra. Claudia Fierro Monti

Depto. de Pediatría Bucal

Facultad de Odontología

Universidad de Concepción

Concepción - CHILE

Email: posgradofao@udec.cl

Recibido : 15-12-2009

Aceptado: 20-04-2010 